\title{
Behandlungsmöglichkeiten des Magenkarzinoms mit Irinotecan
}

\author{
Irinotecan - Therapeutic Options in the Treatment of Gastric Cancer
}

\author{
C.-H. Köhne \\ Hämatologie/Onkologie, Universität Rostock
}

Das Magenkarzinom ist weltweit einer der häufigsten Tumoren. E s ist bei $\mathrm{M}$ ännern für $10 \%$ und bei Frauen für $6 \%$ aller malignen Erkrankungen verantwortlich. Weltweit erkranken jährlich etwa 1 M illion M enschen an diesem Tumor, in Deutschland liegt die jährliche Inzidenz im Bereich von 30-35 Neuerkrankungen pro 100.000. Der Häufigkeitsgipfel des $M$ agenkarzinoms liegt jenseits des 60 . L ebensjahres, wobei 2,2-mal mehr $M$ änner als Frauen betroffen sind.

\section{Status quo}

In der Behandlung des metastasierten Magenkarzinoms werden seit Jahrzehnten die verschiedensten Zytostatika eingesetzt, zumeist aus den W irkstoffgruppen der A ntimetabolite (5-Fluorouracil, M ethotrexat), A ntibiotika (M itomycin C, A nthrazykline) und Platin-K omplexverbindungen. O bgleich ältere U ntersuchungen gelegentlich höhere R emissionsraten ergaben, dürfte unter Zugrundelegung der heutigen strengen R emissionskriterien kaum einer dieser Wirkstoffe in Monotherapie eine Remissionsrate von mehr als $20 \%$ induzieren. Lange Zeit galt es als fraglich, ob Patienten überhaupt von einer Chemotherapie im metastasierten Stadium profitieren können. Inzwischen liegen aber die Daten mehrerer randomisierter Studien vor, die einen signifikanten Ü berlebensvorteil im Chemotherapiearm gegenüber einer alleinigen supportiven Therapie ('best supportive care', BSC) belegen [1, 2]. Der E ffekt war zumeist sehr ausgeprägt. So wurde die mediane Ü berlebenszeit durch die Chemotherapie um den Faktor 2-4 von etwa 3-5 M onaten auf bis zu $12 \mathrm{M}$ onate verlängert. In jüngster Zeit wurde versucht, wirksamere Chemotherapieregime zu entwickeln. Im Vergleich mit dem FA M TX-R egime (5-Fluorouracil, D oxorubicin, M ethotrexat), das in der Vergangenheit beim $M$ agenkarzinom häufig eingesetzt wurde, erwies sich das E CF ${ }_{\mathrm{ci}^{-}}$-Schema mit E pirubicin, Cisplatin und 5-Fluorouracil (letzteres als kontinuierliche Infusion) in einer randomisierten Studie als signifikant wirksamer [3]. D ie R emissionsrate wurde mehr als verdoppelt (45\% vs. $21 \%$ ) und die mediane Ü berlebenszeit von 5,7 auf 8,9 M onate signifikant verlängert. $E$ in daraus abgeleitetes Regime, bei dem Epirubicin durch $M$ itomycin $C$ ersetzt wurde $\left(M C F_{c i}\right)$, zeigte sich in einer randomisierten Studie gleich wirksam wie $E \mathrm{CF}_{\mathrm{c}}$, mit einer A nsprechrate von 39\% und einer medianen Ü berlebenszeit von 8,8 $\mathrm{M}$ onaten [4]. $\mathrm{E}$ inen anderen Weg beschritt die deutsche A rbeitsgruppe am Klinikum E ssen, die ein Hochdosis-Infusionsschema von 5-Fluorouracil/Folinsäure (5-Fluorouracil $2 \mathrm{~g} / \mathrm{m}^{2}$ als 24-h-Infusion wöchentlich $\times 6+$ Folinsäure 500 $\mathrm{mg} / \mathrm{m}^{2}$ wöchentlich $\times 6$ ) mit Cisplatin $50 \mathrm{mg} / \mathrm{m}^{2}$ alle 2 Wochen $\times 3$ kombinierte. $D$ ie Z yklen wurden alle 8 Wochen wiederholt. $B$ ei relativ geringer Toxizität gelangten von den 83 behandelten Patienten $5(6 \%)$ in eine komplette und 33 in eine partielle R emission (G esamtansprechrate $47 \%$ ). D as progressionsfreie Intervall betrug $7 \mathrm{M}$ onate und die mediane $\mathrm{G}$ esamtüberlebenszeit $11 \mathrm{M}$ onate.

$\mathrm{U}$ m den Stellenwert der 5-Fluorouracil-I nfusionsschemata und von Cisplatin beim fortgeschrittenen Magenkarzinom noch genauer zu definieren, führt die EORTC eine randomisierte Studie mit 4 A rmen durch: FAMTX versus 5-Fluorouracil alleine versus 5-Fluorouracil/Folinsäure versus 5-Fluorouracil/ Folinsäure + Cisplatin. D er A rm mit der 5-Fluorouracil-M onotherapie wurde inzwischen geschlossen, da weitgehend auszuschließen war, dass eine R emissionsrate von über $25 \%$ erreicht werden konnte. Die Ergebnisse der anderen A rme werden gegenwärtig ausgewertet.

Der Status quo in der Therapie des metastasierten Magenkarzinoms lässt sich damit wie folgt zusammenfassen: D ie Patienten profitieren von einer systemischen Chemotherapie, wobei 5-Fluorouracil (verabreicht als Dauerinfusion) und Cisplatin offenbar zu den wirksamsten Komponenten der Therapie zählen. Der Stellenwert von A nthrazyklinen und $M$ itomycin $C$ ist noch nicht ausreichend geklärt.

\section{Monotherapiestudien mit Irinotecan}

A ngesichts der nach wie vor unbefriedigenden Behandlungsergebnisse ist die Entwicklung neuer Chemotherapieregime unter $\mathrm{E}$ inbeziehung neuer $\mathrm{E}$ inzelsubstanzen notwendig und wünschenswert. Eine vielversprechende Perspektive bietet hier das I rinotecan. D ie A ktivität der M onosubstanz wurde in einer Phase-II-Studie, an der sich Zentren in mehreren europäischen Ländern und Israel beteiligten, untersucht [5].

\begin{tabular}{ll}
\hline KARGER & ○ 2000 S. Karger G mbH , Freiburg \\
$\begin{array}{l}\text { Fax }+497614520714 \\
\begin{array}{l}\text { E-mail Information@K arger.de } \\
\text { www.karger.com }\end{array}\end{array}$ & $\begin{array}{l}\text { A ccessible online at: } \\
\text { www.karger.com/journals/onk }\end{array}$
\end{tabular}

Dr. Claus-H enning K öhne U niversität R ostock

$\mathrm{H}$ ämatologie/O nkologie

E rnst-H eydermann-Str. 6

D-18055 R ostock (Germany) 
I rinotecan wurde dabei in einer D osierung von $350 \mathrm{mg} / \mathrm{m}^{2}$ alle 3 Wochen eingesetzt. Primärer Studienendpunkt war die objektive R emissionsrate. D ie R emissionen wurden dabei von einem unabhängigen Expertengremium begutachtet. Sekundäre E ndpunkte waren Verträglichkeit, R emissionsdauer und progressionsfreies I ntervall. Insgesamt wurden 40 Patienten in die Studie aufgenommen, bei 34 von innen war das Therapieergebnis auswertbar. D ie B ehandlung mit I rinotecan führte zu 3 kompletten und 4 partiellen Remissionen, was einer Gesamtansprechrate von $20 \%$ entspricht. Doppelt so viele Patienten $(40 \%)$ zogen allerdings einen subjektiven Nutzen aus der Behandlung. Das progressionsfreie Intervall betrug 2,6 M onate, die mediane Ü berlebensdauer 7,5 M onate. Die geschätzte Überlebensrate nach 1 Jahr liegt bei etwa $20 \%$ (A bb. 1).

A us diesen Ergebnissen kann der Schluss gezogen werden, dass I rinotecan zu den wirksamsten Substanzen beim metastasierten M agenkarzinom gehört. Die Behandlung wurde trotz der hämatologischen Toxizität (N eutropenie $\mathrm{G}$ rad $3 / 4$ bei $31 \%$ der Patienten) gut toleriert. Nur in 2 Fällen $(5 \%)$ trat eine febrile Neutropenie auf. Eine Diarrhö Grad 3/4 entwickelte sich bei $18 \%$ der Patienten.

$D$ ie $E$ rgebnisse zur $M$ onoaktivität von I rinotecan stimmen gut mit denen aus Japan und den USA überein. Die japanische A rbeitsgruppe erzielte mit einem wöchentlichen oder zweiwöchentlichen Schema ( $100 \mathrm{mg} / \mathrm{m}^{2} /$ Woche bzw. $150 \mathrm{mg} / \mathrm{m}^{2}$ alle 2 Wochen) bei insgesamt 60 Patienten eine A nsprechrate von $23 \%$ [6]. D ie amerikanische $\mathrm{G}$ ruppe behandelte mit 4 wöchentlichen Dosen von $125 \mathrm{mg} / \mathrm{m}^{2}$ Irinotecan bei anschließender 2-wöchiger Therapiepause und erreichte bei 1 von 7 auswertbaren Patienten eine komplette Remission (14\%) und bei weiteren 5 Patienten (71\%) eine teilweise lang anhaltende Tumorstabilisierung [7].

\section{Kombinationstherapie mit Irinotecan}

A ufgrund dieser E rgebnisse erscheint es sinnvoll, I rinotecan auch in Kombinationsregime zur B ehandlung des Magenkarzinoms zu integrieren. Dabei könnte die Kombination von I rinotecan mit Cisplatin besondere Vorteile bieten. A uch sollte die M öglichkeit verfolgt werden, I rinotecan zusätzlich in das bereits hochaktive Kombinationsregime von Cisplatin plus 5-Fluorouracil/Folinsäure nach dem AIO-Schema zu integrieren.

E ine Phase-I/II-Studie in Japan setzte I rinotecan in einer Dosierung von $70 \mathrm{mg} / \mathrm{m}^{2}$ an Tag 1 und 15 zusammen mit Cisplatin $80 \mathrm{mg} / \mathrm{m}^{2} \mathrm{Tag} 1 \mathrm{mit}$ Zykluswiederholung alle 4 Wochen ein [8]. D osislimitierende Toxizität war die N eutropenie. Insgesamt wurden 24 Patienten in die Studie einbezogen. Von 4 unvorbehandelten Patienten gelangten 3 in eine R emission, von 20 vorbehandelten Patienten (19 mit 5-Fluorouracil, 6 mit Cisplatin) erreichten 7 eine Remission. $D$ as ergibt eine $\mathrm{Ge}$ samtansprechrate von $42 \%$. In der Phase-II-Studie von B oku et al. [9] wurde dieses Kombinationsregime dann bei weiteren 44 Patienten eingesetzt. B ei den 29 chemotherapeutisch unvorbehandelten Patienten wurde eine exzellente Ansprechrate von $59 \%$ erzielt, bei den 15 vorbehandelten Patienten betrug sie $27 \%$. Die Ü berlebensdaten waren ebenfalls vielverspre-
Tab. 1 E rgebnisse der europäischen Phase-II-Studie mit I rinotecan beim metastasierten Magenkarzinom (mod. nach K öhne et al. [5])
A nzahl Patienten / auswertbar:

Vollständige (CR)/partielle (PR) R emission:

Subjektive B esserung:

Progressionsfreies I ntervall:

M edianes Ü berleben:

G esamtüberlebensrate nach 6/9/12 M onaten:
$40 / 34$

$3 C R+4 P R=20 \%$

$40 \%$

2,6 M onate

7,5 M onate

$65 / 39 / 20 \%$ chend. D ie mediane G esamtüberlebensdauer aller 44 Patienten betrug 8,9 $\mathrm{M}$ onate und das mediane Ü berleben der unvorbehandelten Patienten sogar 10,6 M onate. Wiederum standen die hämatologische Toxizität (Leukopenie, Neutropenie, A nämie) und die Diarrhö im Vordergrund.

A uf dem A SCO-Kongress 1999 wurden die Ergebnisse von zwei Studien amerikanischer A rbeitsgruppen vorgestellt, in denen ein Schema mit 4 wöchentlichen D osen von Irinotecan/ Cisplatin und anschließender 2-wöchiger Therapiepause angewandt wurde. In der ersten Studie wurden bislang lediglich 11 auswertbare Patienten mit I rinotecan $65 \mathrm{mg} / \mathrm{m}^{2} /$ Woche und Cisplatin $30 \mathrm{mg} / \mathrm{m}^{2} /$ Woche behandelt, von denen 2 (18\%) in eine partielle Remission gelangten [10]. Die Rekrutierung von Patienten für diese Studie wird fortgesetzt. In die zweite Studie wurden auch vorbehandelte Patienten aufgenommen, bei innen wurde die wöchentliche I rinotecan-D osis aber von 65 auf $50 \mathrm{mg} / \mathrm{m}^{2}$ reduziert [11]. Interessanterweise war die A nsprechrate in beiden Teilkollektiven dieser Studie ganz ähnlich, 51\% (10 PR +3 CR ) bei den 25 unvorbehandelten Patienten und $50 \%$ ( 4 PR) bei den 8 vorbehandelten Patienten. $\mathrm{D}$ a trotz des E insatzes von G-CSF bei 17 Patienten die Toxizität beträchtlich war und häufig $D$ osen ausgelassen werden mussten, sehen die A utoren eine M odifikation dieses Schemas für sinnvoll an.

\section{Ausblick}

D ie guten B ehandlungsergebnisse mit I rinotecan-K ombinationen beim fortgeschrittenen $M$ agenkarzinom lassen es sinnvoll erscheinen, ihren therapeutischen Stellenwert in randomisierten Studien definitiv zu klären. A ngelaufen ist in D eutschland eine Phase-II/III-Studie, in der zunächst in einem vorgeschalteten randomisierten Phase-II-A bschnitt jeweils 45 Patienten entweder mit der Kombination Irinotecan/Cisplatin oder der Kombination I rinotecan/5-Fluorouracil (nach dem A IO -Schema) behandelt werden. $D$ as bessere dieser beiden Kombinationsregime - wobei neben der Wirksamkeit auch die A nwendungssicherheit Berücksichtigung findet - wird anschließend in einer Phase-III-Studie mit der Kombination Cisplatin/ 5-Fluorouracil verglichen. Geplant ist hierfür eine R ekrutierung von jeweils 171 Patienten pro A rm. Primärer E ndpunkt ist das progressionsfreie Intervall, sekundäre Endpunkte sind A nsprechrate, Remissionsdauer, Z eit bis zum Therapieversagen, L ebensqualität und Gesamtüberleben. Der Phase-IIA bschnitt dieser Studie wird in Kürze abgeschlossen werden, so dass der Phase-III-Vergleich anlaufen kann. 


\section{Literatur}

1 M urad A M, Santiago FF, Petroianu A, R ocha PR, Rodrigues MA, Rausch $M$ : Modified therapy with 5-fluorouracil, doxorubicin, and methotrexate in advanced gastric cancer. Cancer 1993;72:37-41.

2 Pyrhönen $S$, Kuitunen $T$, Nyandoto $P$, Kouri $M$ : $R$ andomised comparison of fluorouracil, epidoxorubicin and methotrexate (FEMTX) plus supportive care with supportive care alone in patients with non-resectable gastric cancer. Br J Cancer 1995;71: 587-591.

3 Webb $A$, Cunningham $D$, Scarffe $J H, H$ arper $P$, Norman A, J offe J K, H ughes M, M ansi J, Findlay M $H$ ill $A, O$ ates J, Nicolson $M, H$ ickish $T, O$ 'B rien $M$, I veson $T$, Watson $M$, U nderhill $C$, Wardley $A$, M eehan $M: R$ andomized trial comparing epirubicin, cisplatin, and fluorouracil versus fluorouracil, doxorubicin, and methotrexate in advanced esophagogastric cancer. I Clin O ncol 1997;15:261-267.
4 Ross $P$, Cunningham $D$, Scarffe $H, N$ icolson $M$ Seymour $M, H$ arper $P$, Price $T, H$ ill $A$, A nderson $H$, I veson $T, H$ ickish T, L ofts $F, N$ orman $A$ : R esults of randomised trial comparing ECF with $M C F$ in advanced oesophago-gastric cancer. Proc A m Soc Clin Oncol 1999;18:1042(abstr).

$5 \mathrm{~K}$ ohne $\mathrm{CH}$, Thuss-Patience $\mathrm{P}$, Catane $\mathrm{R}, \mathrm{K}$ lein $\mathrm{H}$ Peretz T, Preusser P, Niederle N, D ucreux M, Wilke H, Jacques $C$ : Final results of a phase II trial of CPT-11 in patients with advanced gastric cancer. Proc A m Soc Clin O ncol 1999;18:993(abstr).

6 Futatsuki K, Wakui A, Nakao I, Sakata Y, K ambe M, Shimada $Y, Y$ oshino $M$, Taguchi T, O gawa N: L ate phase II study of irinotecan hydrochloride (CPT-11) in advanced gastric cancer. CPT-11 Gastrointestinal Cancer Study Group. Gan To Kagaku Ryoho 1994;21:1033-1038.

7 Hecht JR, Parson M, R osen LS: A phase II trial of irinotecan (CPT-11) in patients with adenocarcinoma of the esophagus and gastric cardia. ProcA $m$ Soc Clin Oncol 1999;18:1100(abstr).
9 B oku N, O htsu A , Shimada Y, Shirao K, Seki S, Saito $\mathrm{H}$, Sakata $\mathrm{Y}, \mathrm{H}$ yodo I: Phase II study of a combination of irinotecan and cisplatin against metastatic gastric cancer. J Clin O ncol 1999;17:319-323.

8 Shirao K, Shimada Y, Kondo H, Saito D, Yamao T, O no H, Y okoyama T, Fukuda H, O ka M, Watanabe Y, O htsu A, B oku N, Fujii T, O da Y, M uro K, Y oshida S: Phase I-II study of irinotecan hydrochloride combined with cisplatin in patients with advanced gastric cancer. J Clin Oncol 1997;15:921-927.

10 Ilson D, E nzinger P, Saltz L, R eilly E O, Schwartz G, Sharma S, G ollub M, H uang Y, G onzalez G, K elsen $D$ : Phase II trial of weekly irinotecan + cisplatin in advanced gastric cancer. Proc A m Soc Clin Oncol 1999;18:994(abstr).

11 A jani JA, Fairweather J, Pisters PW, Feig BW, $\mathrm{H}$ argraves K, Charnsanngavej C: Phase II study of CPT-11 plus cisplatin in patients with advanced gastric and GE junction carcinomas. Proc A m Soc Clin O ncol 1999;18:927(abstr). 